\title{
Isolating nanoparticles from complex biological media by immunoprecipitation
}

Youcef Chakib Hacene', Alexis Loiseau², Vanessa Maria Dos Passos Maio', Philippe

$$
\text { Grenier }^{1} \text {, Elodie Boisselier2, Nicolas Bertrand }{ }^{1 *}
$$

${ }^{1}$ Faculty of Pharmacy, CHU de Quebec Research Center, Université Laval, CHUL,

2705 Laurier Blvd, Québec G1V 4G2, Canada

${ }^{2}$ Faculty of Medicine, Department of Ophthalmology, CHU de Québec Research

Center, Université Laval, Hôpital du Saint-Sacrement, CUO-Recherche, 1050, chemin

Sainte-Foy, Québec, G1S 4L8, Canada

\section{Materials and methods}

$\mathrm{N}$-(terbutoxycarbonyl)-ethanolamine (tBoc-ethanolamine) and triazabicyclodecene

(TBD) were purchased from Sigma Aldrich (Saint Louis, USA). DL-lactide (Acros

Organics, Fisher Scientific, California, USA) was crystallized twice from ethyl acetate and dried under reduced pressure. Trifluoroacetic acid (reagent grade) and solvents were 
obtained from Fisher Scientific (Massachusetts, USA). N-hydroxysuccinimide esters of borondipyrromethene R6G (BDP(R6G), product \# 44420) and cyanine 5.5 (Cy5.5, product \# 47020) were purchased from Lumiprobe (Florida, USA).

\section{Synthesis of borondipyrromethene and cyanine terminated polymers.}

Poly(lactic acid) polymers with a primary amine terminal groups were synthesized by adapting a ring-opening polymerization method ${ }^{26}$. Briefly, tBoc-ethanolamine $(0.09 \mathrm{mmol}$, 1 eq) was dissolved under an inert atmosphere in $6 \mathrm{~mL}$ of anhydrous dichloromethane containing DL-lactide $(13.2 \mathrm{mmol}, 147 \mathrm{eq})$. The catalyst, TBD $(0.135 \mathrm{mmol}, 1.5 \mathrm{eq})$ was dissolved in $0.1 \mathrm{~mL}$ of dichloromethane and added to the reaction. The mixture was polymerized at room temperature for 30 minutes and quenched by addition of benzoic acid $(0.7 \mathrm{mmol}, 6.5 \mathrm{eq})$. The polymer solution was concentrated by rotary evaporation and precipitated twice in cold diethyl ether.

The terminal amine was deprotected by removal of the protecting group in $6 \mathrm{~mL}$ anhydrous dichloromethane containing $2 \mathrm{~mL}$ of trifluoroacetic acid at $0{ }^{\circ} \mathrm{C}$ for 1 hour. The solution was concentrated and precipitated twice in cold diethyl ether. An excess of 
amine-terminated PLA was reacted with the dyes (BDP(R6G)-NHS or Cy5.5-NHS) at room temperature. Briefly, $5 \mathrm{mg}$ of dye $(0.015 \mathrm{mmol}$ of BDP(R6G)-NHS, or $0.006 \mathrm{mmol}$ of Cy5.5-NHS, $1 \mathrm{eq})$ and $100 \mathrm{mg}$ of PLA $(0.07 \mathrm{mmol}, 2$ or $10 \mathrm{eq})$ were dissolved in 0.4 $\mathrm{mL}$ of anhydrous dichloromethane and protected from light. Anhydrous disopropylethylamine $(200 \mu \mathrm{L}, 1.15 \mathrm{mmol})$ was added, and the conjugation was conducted at room temperature for 18 hours. The fluorescent polymer was recovered by two precipitations in cold methanol and dried overnight under vacuum. 


\section{PEG-PLGA nanoparticles preparation}

PEG-PLGA nanoparticles were prepared by nanoprecipitation as described

previously[1, 2]. Briefly, $5 \mathrm{mg} / \mathrm{mL}$ solutions of copolymers in acetonitrile were added dropwise to $10 \mathrm{~mL}$ of water under magnetic stirring in a $20 \mathrm{~mL}$ vial ${ }^{10}$. The polymer mixtures used in the preparation of nanoparticles are described in Supplementary Table 1. Nanoparticles were labeled with a radioactive $\left[{ }^{14} \mathrm{C}\right]-\mathrm{PLGA}$ copolymer $(\mathrm{Mn} \sim 20,000$, Moravek Biochemicals) or with a fluorescent [Cy5.5]-poly(lactic acid) polymer (synthesized as described in supplementary methods). For all experiments, nanoparticles were purified and washed once with a 10:90 acetonitrile:water solution, then 5 times with ultrapure water using an ultrafiltration filter (PALL MicroSep MCWO 100,000). The average diameter (Z-average) and polydispersity index (PDI) of the nanoparticles were measured before and after purification by dynamic light scattering method (DLS) at $22^{\circ} \mathrm{C}$ with a $173^{\circ}$-backscatter angle on a Malvern Zetasizer Nano S (Malvern Instruments, Westborough, MA). Structural characterization of the outside shell and PEG content of nanoparticles were determined by ${ }^{1} \mathrm{H}$ NMR spectroscopy, as described previously [2]. 


\section{Liposomes preparation and quantification}

PEGylated Liposomes were prepared by the hydration of lipid films followed by extrusion through polycarbonate membranes[3]. Hydrogenated phosphatidylcholine (> 98\% pure, Coatsome NC-21E, NOF American Corporation, White Plains, NY), cholesterol (NF grade, Medisca, Montreal, Canada), 14C-cholesterol oleate (Perkin Elmer, Waltham, $\mathrm{MA}$ ), and a $5 \mathrm{~mol} \%$ of $\mathrm{PEG}_{2 \mathrm{k}}$-distearoyl-glycero-phosphoethanolamine (Sunbright $\AA$ DSPE-020CN, NOF American Corporation, White Plains, NY) were dissolved in dichloromethane. For liposomes containing Vitamin K (Vitamin K1, Alfa Aesar, USA), 10 $w t \%$ of the drug were added to the organic phase. The organic solvent was evaporated in a rotary evaporator [4].

The thin lipid film was hydrated with 4-(2-hydroxyethyl)-1-piperazineethanesulfonic acid (HEPES) saline buffer (20 mM HEPES, and $140 \mathrm{mM} \mathrm{NaCl}, \mathrm{pH}$ 7.4) under constant stirring and periodic sonication for 24 hours and then extruded using LipoFast $₫$ manual extruder (Avestin, Ottawa, ON, Canada) through 200, 100 and $50 \mathrm{~nm}$ polycarbonate membranes. Liposomes containing 8-Hydroxypyrene-1,3,6-trisulfonate (HPTS) (Fisher Scientific 
(Tewsbury, MA, USA)) and p-Xylene-bis(N-pyridinium bromide) (DPX) (Fisher Scientific (Tewsbury, MA, USA)) were prepared by hydrating the lipid film with an aqueous solution containing $18.7 \%$ and $24.6 \% \mathrm{wt} \%$ of HPTS and DPX, respectively. Non-encapsulated dye was removed by size-exclusion chromatography using a $1 \times 10 \mathrm{~cm}$ gravity-flow column (Kimble-Chase gravity Flexcolumn, \#420401-1010, Vineland, NJ, USA)) loaded with Sepharose CL-4B gel (GE Healthcare (USA)). Liposomes formulations were characterized by dynamic light scattering and a colorimetric phosphorous assay to determine concentration [5].

\section{Immunoprecipitation procedure}

Magnetic immunoprecipitation beads (Protein G Dynabeads, Thermo Fisher Scientific,

USA) and antiPEG (Recombinant Anti-Polyethylene glycol antibody R105, product \# ab190652, Abcam, MA, USA) or control isotype antibodies (recombinant rabbit monoclonal IgG EPR25A, product \#ab172730, Abcam, MA, USA) were used in all immunoprecipitation procedures. In all experiments, the ratio between the volume of 
magnetic beads and the quantity of antibodies was kept constant at $25 \mu \mathrm{L}$ of beads (750

$\mu g)$ for $5 \mu g$ of antibody.

Incubation procedures: To extract PEGylated nanoparticles from $50 \mu \mathrm{L}$ of phosphate buffered saline (PBS), nanoparticles were preincubated with antibodies for 2 hours under constant stirring. Next, the nanoparticles/antibodies complexes were added to the corresponding amount of magnetic beads and incubated for 24 hours at $4{ }^{\circ} \mathrm{C}$, with regular manual mixing using a $200 \mu \mathrm{L}$ pipette, unless specified otherwise. A 6-tube magnetic separation rack (New England Biolabs, USA) was used to isolate magnetic beads from the supernatant containing non-extracted nanoparticles.

For experiments involving extraction from plasma, nanoparticles were incubated ex vivo for 30 minutes in $50 \mu \mathrm{L}$ of rat plasma (Sterile rat plasma GTX73218, GeneTex, USA) or injected to live animals prior to separation. Plasma-coated nanoparticles were incubated with freshly crosslinked magnetic beads (see below for crosslinking procedure) for 24 hours at $4{ }^{\circ} \mathrm{C}$, with regular manual mixing using a $200 \mu \mathrm{L}$ pipette. 
For experiments involving extraction from human biological media, nanoparticles were dispersed in vitro into $50 \mu \mathrm{L}$ of pooled human urine, pooled human tears, pooled human sweat and pooled human saliva (all obtained from Innovative Research, USA). Human aqueous humor and human vitreous humor were obtained from human eyes from our local Eye Bank (Banque d'Yeux du Centre Universitaire d'Ophthalmologie, Quebec City, Quebec, Canada). Human vitreous humor was heated to a temperature of $41^{\circ} \mathrm{C}$ before it was mixed with nanoparticles and kept at that temperature during the whole extraction process. The extraction was conducted for 2 hours and the magnetic beads dispersion was mixed using a pipette at 20 minutes time intervals.

For experiments using solutions of proteins, bovine serum albumin (Sigma) or recombinant mouse Apolipoprotein E (His tag) (ab226314, Abcam), were dissolved in PBS and used.

Washing and elution: Magnetic beads isolated from the aforementioned media were washed twice with PBS to eliminate weakly-adsorbed nanoparticles. Nanoparticles were eluted (desorbed) from the beads using a $0.1 \mathrm{M}$ citric acid buffer $(\mathrm{pH} 2.4)$, or by incubating 
for 15 minutes at room temperature in a $1 \%$ sodium dodecyl sulfate (SDS) solution. The former elution buffer was used to desorb nanoparticles from microbeads non-crosslinked with antibodies, and the second to elute nanoparticles from microbeads which were crosslinked.

The content in radioactive nanoparticles was assessed in all media by scintillation counting on a Hidex 300 SL TDCR LSC (Hidex, Finland), by addition of $5 \mathrm{~mL}$ of Cytoscint Liquid Scintillation Cocktail (MP Biomedicals, Solon, OH, USA). Protein content in the supernatant was measured using the $\mathrm{BCA}$ or the micro $\mathrm{BCA}$ protein assay (Thermo Fisher Scientific, USA), using a calibration curve of bovine serum albumin.

\section{Calculation of extraction percentages and extraction capacities.}

The percentage of radioactivity (Figure 1 and $2,5 \mathrm{~A}$ ) is the ratio of the radioactivity in the sample over the total radioactivity at the beginning of the experiment. The percentage of radioactivity extracted (Figure $3 \mathrm{~A}$ ) is $100 \%$ minus the percentage of radioactivity recovered in the supernatant. Quantity extracted (Figure S1A) is the percentage of radioactivity extracted multiplied by the concentration (in mass) of the initial sample, as 
determined by gravimetry. The quantity eluted (Figures $\mathrm{S} 1 \mathrm{~A}$ ) is calculated from the mass concentration, using the percentage of radioactivity in the elution buffer. The extraction capacity (Figure 3B) is the maximal quantity extracted using $25 \mu \mathrm{l}$ of magnetic beads (i.e., $750 \mu \mathrm{g}$ of solid). Some extraction capacities are reported as percentages of the maximum extraction capacity measured in these conditions (Figure S1B, S2, S3, and S8). The number of nanoparticles (Figure S6) is calculated as detailed in [2].

\section{Crosslinking of magnetic beads with antibodies}

One typical batch of crosslinked Dynabeads consisted of $25 \mu \mathrm{L}$ of magnetic beads and $5 \mu \mathrm{g}$ of antibodies. Magnetic beads were incubated with antibodies for at least 2 hours under regular stirring at $4{ }^{\circ} \mathrm{C}$, then washed twice with $125 \mu \mathrm{L}$ of conjugation buffer $(20$ $\mathrm{mM} \mathrm{NaH}{ }_{2} \mathrm{PO}$, $150 \mathrm{mM} \mathrm{NaCl}, \mathrm{pH}$ 7.5). Crosslinking was induced by addition of $125 \mu \mathrm{L}$ of a freshly prepared $5 \mathrm{mM}$ solution of bis(sulfosuccinimidyl)suberate (BS3, Thermo Fisher Scientific, USA) in conjugation buffer. After reacting for 45 minutes at room temperature with regular manual mixing, the reaction was quenched with $6.25 \mu \mathrm{L}$ of a $1 \mathrm{M}$ tris(hydroxymethyl)aminomethane solution ( $\mathrm{pH}$ 7.5). After 15 minutes of quenching, 
dynabeads were washed 2 times with phosphate buffered saline to remove unreacted products and antibodies.

\section{In vivo experiments}

All animal studies were conducted using institutionally approved protocols at Université

Laval (Canadian Council on Animal Care standards and Animal Research: Reporting In

Vivo Experiments guidelines). Healthy animals (male Balb/c mouse, 23-25 g) were

housed in a controlled environment $\left(22^{\circ} \mathrm{C}, 12 \mathrm{~h}\right.$ day/night cycle) with ad libitum access

to food and water.

One hundred $\mu \mathrm{L}$ of a $5 \mathrm{mg} / \mathrm{mL}$ suspension of nanoparticles, dual-labeled with ${ }^{14} \mathrm{C}-\mathrm{PLGA}$ and BDP(R6G-)-PLA (synthesis procedure in supplementary material), were injected via the intravenous or intraperitoneal routes. Intravenous injections were done in the subclavian vein, under isoflurane anesthesia. At predefined time points, blood was collected in microcentrifuge tubes containing $100 \mu \mathrm{L}$ of a $340 \mathrm{mM}$ ethylenediaminetetraacetic acid (EDTA), by cardiac puncture under isoflurane anesthesia. Plasma was separated by centrifuging blood for 10 minutes at 3000 rcf. The 
concentration of nanoparticles in plasma was measured by scintillation counting, as described. For both administration routes, plasma containing $15 \mu \mathrm{g}$ of nanoparticles was incubated with $25 \mu \mathrm{L}(750 \mu \mathrm{g})$ of magnetic beads crosslinked with $5 \mu \mathrm{g}$ of antiPEG antibodies and eluted as described above.

After elution, the size of nanoparticles was determined using the Zetaview Fluorescence Nanoparticle Tracking Analysis (F-NTA, Particle Metrix, Germany) equipped with a 520 $\mathrm{nm}$ laser, and a broad-pass filter. Samples were diluted in water to a concentration of approximately $4 \times 10^{7}$ particles $/ \mathrm{mL}$. Camera settings were: Sensitivity 90 , Shutter speed 62, Highest resolution, 60 frames/sec. Software settings were: Minimum brightness 40 , Minimum area 20, Maximum area 1000, Trace length 10. With control batches of nanoparticles, these settings were shown to minimize differences between the median obtained by F-NTA and the Z-average obtained by DLS.

\section{Centrifugation experiments}

Rat plasma (Sterile rat plasma GTX73218, GeneTex, USA) was first centrifuged during 10 minutes at $15000 \mathrm{RCF}$ and $4{ }^{\circ} \mathrm{C}$. PLGA-PEG nanoparticles and liposomes were 
dispersed in $500 \mu \mathrm{L}$ of rat plasma, and centrifuged during 20,60 or 120 minutes. At the end of the first centrifugation cycle, the pellet was washed twice using 500 ul PBS by mixing using a pipette and a vortex, then centrifuged for the same duration at the same speed (15000 RCF) and temperature $\left(4^{\circ} \mathrm{C}\right)$. At the end of the 3 centrifugations, the pellet is redispersed in PBS and analyzed in terms of radioactivity to determine the quantity of sedimented nanoparticles and using BCA or micro BCA to determine the quantity of sedimented proteins. Plasma without nanoparticles was also included as a negative control.

\section{Preparation of vitamin K loaded PLGA-PEG nanoparticles}

PLGA-PEG nanoparticles loaded with vitamin K (Vitamin K1, Alfa Aesar, USA) were prepared via nanoprecipitation. Vitamin K was dissolved with 80\% PLGA21k-PEG5k and $20 \%$ PLGA95k in acetonitrile. The organic solution is added to ultrapure water drop-wise under constant agitation at $1600 \mathrm{rpm}$. Nanoparticles are purified using Strata ${ }^{\mathrm{TM}}-\mathrm{X} 33 \mu \mathrm{m}$ Polymeric Reversed Phase column (Phenomenex, USA) then washed 5 times with ultrapure water using an ultrafiltration filter (PALL MicroSep MCWO 100,000). 


\section{Quantification of vitamin K in nanoparticles after exposure to plasma}

Vitamin K loaded PEG-PLGA nanoparticles were incubated in $40 \mu \mathrm{L}$ of rat plasma or HEPES, at a concentration of $0.2 \mathrm{mg} / \mathrm{mL}$, at room temperature under constant stirring at $800 \mathrm{rpm}$. Volumes containing $10 \mu \mathrm{g}$ of nanoparticles were sampled in triplicates after 30 , 60 and 120 minutes and immediately incubated with $750 \mu \mathrm{g}$ magnetic beads prepared with crosslinked antiPEG antibodies as described previously, for 90 minutes. As a negative control, a solution of vitamin $\mathrm{K}$ in plasma was prepared and incubated with magnetic beads bearing antiPEG antibodies.

Vitamin $\mathrm{K}$ was quantified in the initial PLGA-PEG dispersion in plasma, in the incubation supernatant, in the PBS wash, and in the $1 \%$ SDS eluate, via reverse phase chromatography using an Agilent 1260 Infinity II LC System. The mobile phase consisted of a $49 \% / 44 \% / 7 \%$ mixture of acetonitrile, THF, and water respectively. Vitamin $\mathrm{K}$ absorbance was detected at $245 \mathrm{~nm}$.

Investigation of the stability of fluorescent liposomes using size exclusion chromatography 
The effect of the eluting solution on the stability of liposomes was investigated using liposomes loaded with HPTS and DPX. With these liposomes, the fluorescence of HPTS increases drastically, when the vesicles are disrupted and the dye is separated from the DPX quencher [6]. Briefly, purified liposomes were incubated with different concentrations of SDS or mPEG2k (Sigma, USA) dissolved in isotonic HEPES buffer saline 1x. Twenty $\mu$ l of liposomes were added to $20 \mu$ l of SDS or $\mathrm{MPEG}$, to reach final elutant concentrations of $1 \%, 0.5 \%, 0.25 \mathrm{wt} \%$ for SDS and $1 \mathrm{wt} \%$ for $\mathrm{mPEG}$. After 15 minutes of incubation, the solution was loaded on a gravity flow size-exclusion column $(1 \times 10 \mathrm{~cm}$, packed with Sepharose CL-4B), and fractions of $500 \mu \mathrm{L}$ were collected. Fluorescence in each fraction was measured ( $\left.\lambda_{\text {ex/em: }}: 452 / 512 \mathrm{~nm}\right)$.

\section{Cell internalization of native and eluted nanoparticles}

Nanoparticles were synthesized as described above, using PLA-Cy5.5 for fluorescence labelling. Nanoparticles were extracted from PBS and eluted using $0.5 \mathrm{wt} \%$ SDS (as described above). After extraction, the fluorescence was normalized between control (non-extracted) and eluted particles using a calibration curve. U87-MG cells were cultured 
at $37^{\circ} \mathrm{C}$ in Eagle's Minimum Essential Medium (Wisent) supplemented with $10 \%$ fetal bovine serum (Wisent) and 1\% antibiotics (penicillin/streptomycin) (Millipore Sigma), in a 5\% CO2 incubator. U87-MG cells were plated at a density of 100,000 cells/ well in 12well plates the day before the internalization experiment. The day of the internalization experiment, nanoparticles were incubated with the cells for 2 hours. For flow cytometry, cells were trypsinized with $0.05 \%$ Trypsin/0.53mM EDTA for 5 min at $37^{\circ} \mathrm{C}$, resuspended with EMEM, washed once with PBS, once with FACS buffer (PBS with $2 \%$ FBS and1mM EDTA) and then resuspended in 300uL FACS buffer. Cell internalization was measured by flow cytometry on a BD LSR/LSRII (BD Biosciences, Mississauga, ON, CA) using the $640 \mathrm{~nm}$ laser and the 720/60 nm filter. The data were analyzed using the FlowJo 10 software (Tree Star Inc., BD, Ashland, OR, USA).

\section{Proteomic analysis of the protein corona}

In vivo procedure: As described above (in vivo experiments), radiolabeled PLA-PEG nanoparticles were injected IV to male Balb/c mice. Five minutes after administration, 
mice were euthanized under deep isoflurane anesthesia, and blood collected by cardiac puncture in centrifuge tubes containing $100 \mu \mathrm{L}$ of a $340 \mathrm{mM}$ EDTA. Plasma was isolated and used immediately for extraction.

Extraction, elution and removal of the polymer: The plasma samples from each mouse were extracted using both antiPEG antibodies and isotype control antibodies. This allowed to control for non-specific adsorption of proteins on magnetic beads. Nanoparticles were eluted from magnetic beads using sodium deoxycholate $1 \%$ at $95^{\circ} \mathrm{C}$ for 15 minutes. Eluted proteins were separated from polymers by adding a 14-fold equivalent volume of acetonitrile and stored at $-20^{\circ} \mathrm{C}$ overnight after being thoroughly vortexed. Samples were then centrifuged at $20000 \mathrm{rcf}$ during 90 minutes at $-16{ }^{\circ} \mathrm{C}$ to -20 ${ }^{\circ} \mathrm{C}$. Following centrifugation, $1400 \mu \mathrm{l}$ od supernatant was cautiously sampled without disturbing the pellet and $400 \mu \mathrm{l}$ of fresh acetonitrile was added to the tube. After vortexing, the acetonitrile containing the proteins was stored at $-20^{\circ} \mathrm{C}$ for one hour and the tubes were centrifuged again as previously. The remaining acetonitrile is carefully removed and the tubes are left to dry for 4 hours at $60^{\circ} \mathrm{C}$ to yield a white protein precipitate. 
Sample preparation for proteomics: The dried protein pellets were resuspendended in $30 \mu \mathrm{L}$ of ammonium bicarbonate $50 \mathrm{mM}$, sodium deoxycholate $1 \%$ and reduced by DTT $0.2 \mathrm{mM}$ and alkylated with iodoacetamide $0.8 \mathrm{mM}$. Proteins were then digested by overnight incubation with $0.26 \mu \mathrm{g}$ Trypsin at $37^{\circ} \mathrm{C}$. Resulting peptides were purified using C18 StageTips and dried on a Speed Vac. Samples were resuspended in $15 \mu \mathrm{L} L C$ loading solvent and peptide quantities assessed by $205 \mathrm{~nm}$ Nanodrop absorbance measurement. Sample volumes were adjusted in order to inject 1ug of material.

Liquid chromatography Mass spectrometry/Mass spectrometry (LC-MSMS) analysis: Analysis was done on an Orbitrap Fusion Tribrid instrument (Thermo). Runs of 120 min (90 min gradients) were used and the MS was operated in DDA mode.

Data analysis: Only summary analysis is presented in this manuscript (to establish the proof-of-concept), more in-depth analysis will be published in a follow-up paper). Identification and LFQ quantification were done using MaxQuant software v1.6.10.43, and the database was Uniprot Mus musculus reference proteome UP000000589 2020/07/05. Trypsin/P enzyme parameter was selected with two possible missed 
cleavages. Cystein carbamidomethylation was set as a fixed modification while methionine oxidation and protein $\mathrm{N}$-terminus acetylation were set as variable modifications. Mass search tolerance were $5 \mathrm{ppm}$ and $0.5 \mathrm{Da}$ for MS and MS/MS respectively. For protein validation, a maximum False Discovery Rate of $1 \%$ at peptide and protein level was used based on a target/decoy search. MaxQuant was also used for Label Free Quantification. The 'match between runs' option was used with 20 min value as alignment time window and 0.7 min as match time window. Only unique and razor peptides were used for quantification. RStudio 1.2.5033 was used for data processing. Maxquant LFQ normalized intensities were considered for the statistical analysis. When protein intensity values were missing, they were replaced by a noise value corresponding to $1 \%$ percentile of the normalized value for each sample. A protein was considered as quantifiable only if all samples in at least one of the two conditions presented an intensity value and if a minimum of two peptides were assigned. The ratio of the intensity measured in the antiPEG sample (i.e., with nanoparticles) over the control sample (i.e., nonspecifically adsorbed proteins) was calculated. To determine statistically variant proteins, 
an intensity ratio, a limma q-value (Benjamin Hochberg correction) was calculated and considered significant for values $<0.05$.

For the emPAI protein abundance estimation[7], MS/MS peak lists were generated using Thermo Proteome Discoverer version 2.3.0.523 with default parameters (Thermo Scientific). MGF sample files were then analyzed using Mascot (Matrix Science, London, UK; version 2.5.1). Protein validation was carried out on Scaffold 4.8.4 (Proteome Software Inc., Portland, OR) with a less than 1\% FDR target on both the protein and peptide level. Protein abundance estimation was determined with the protein coveragebased emPAl value. Proteins reported on Figure $5 \mathrm{C}$ correspond to all proteins with emPAI values $>4.0$. 


\section{Statistical analysis}

Statistics were computed using GraphPad Prism 8.0. Standard unpaired t-test was used

to compare the means of each group. Data in figure 7A was analyzed via one-parameter ANOVA, using a Tukey post-hoc test. Significance was considered for $p$-values inferior to 0.05 .

\section{Supplementary figures}

\section{A. Using SDS 1\%, the nanoparticles extracted can be eluted quantitatively}

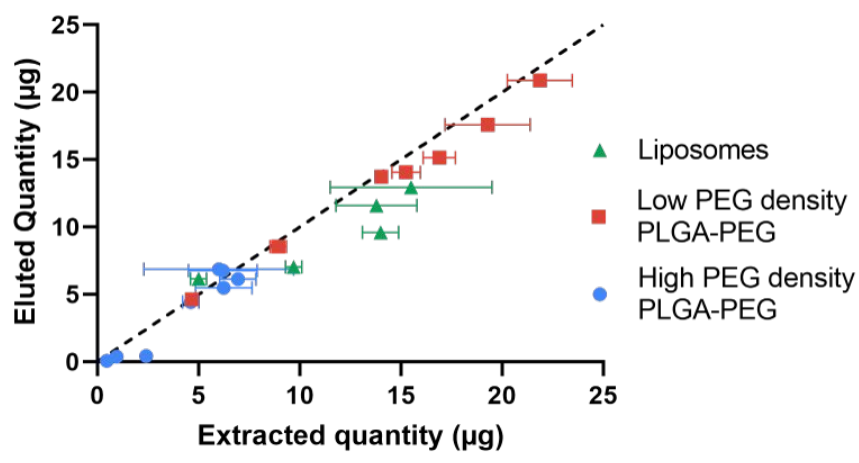

B. Other buffers can be used to efficiently recover the extracted nanoparticles

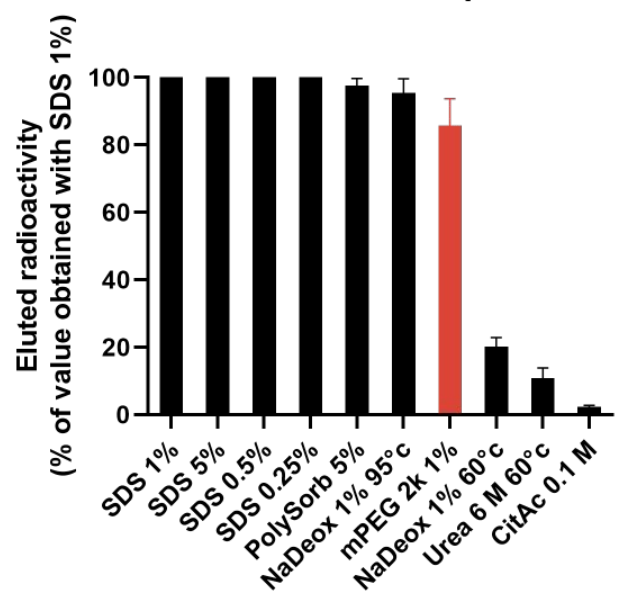

Figure S1. SDS $1 \%$ and other elution buffers can be used to recover extracted nanoparticles from crosslinked magnetic beads in a quantitative manner. A. Various quantities of PEGylated nanoparticles and liposomes can be quantitatively recovered using SDS $1 \mathrm{wt} \%$. B. Low concentrations of surfactants (SDS, sodium deoxycholate) and free $\mathrm{mPEG}$ are able to elute nanoparticles from the magnetic beads. Tested elution buffers from left to right are: Sodium dodecyl sulfate (SDS) 1\%, SDS 5\%, SDS 0.5\%, SDS $0.25 \%$, Polysorbate $5 \%$, Sodium Deoxycholate $1 \%$ at $95{ }^{\circ} \mathrm{C}, \mathrm{mPEG} 2 \mathrm{k} 1 \%$, Sodium 
Deoxycholate $1 \%$ at $60{ }^{\circ} \mathrm{C}$, Urea $6 \mathrm{M}$ at $60{ }^{\circ} \mathrm{C}$, and citric acid $0.1 \mathrm{M}$. Values represent means $+/-S D(n \geq 3)$. 
A. Effect of crosslinking and incubation in plasma on the capacity of extraction

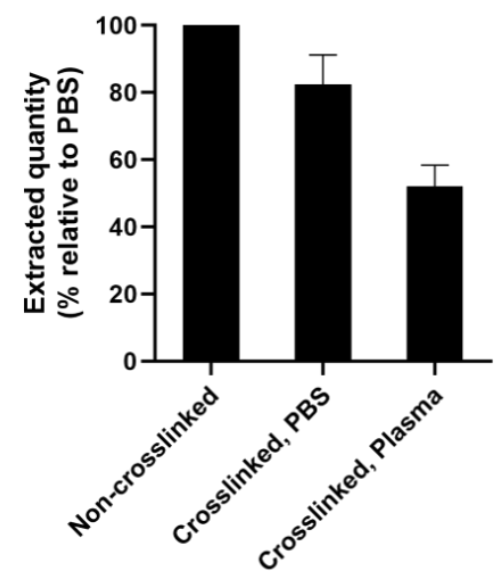

B. Effect of individual proteins on the capacity of extraction

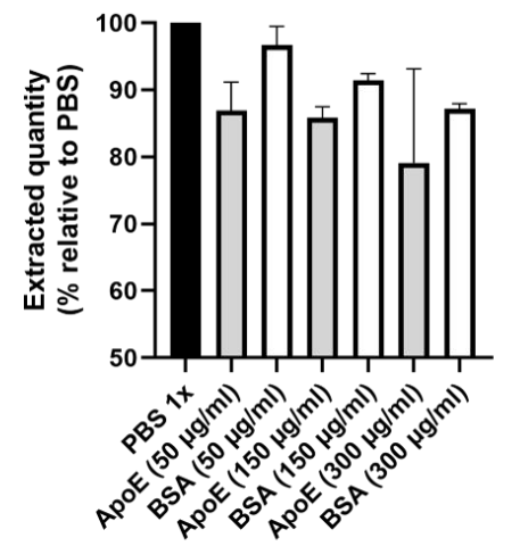

Figure S2. Incubation of crosslinked magnetic beads with plasma and protein solutions show that adsorption of proteins decreases the capacity of magnetic beads. A. Crosslinking and incubation in plasma both decrease the capacity of the extraction process. B. The decrease in capacity observed after the addition of individual proteins (Bovine serum albumin (BSA) or Apolipoprotein E (ApoE) to PBS, show that interactions between the proteins and the magnetic beads or the nanoparticles can alter the extraction process. Nevertheless, in all conditions tested, values remain $\geq 80 \%$ of the capacity measured in PBS. Values represent means $+/-$ SD $(n=3)$

A. Reutilization of magnetic beads in PBS

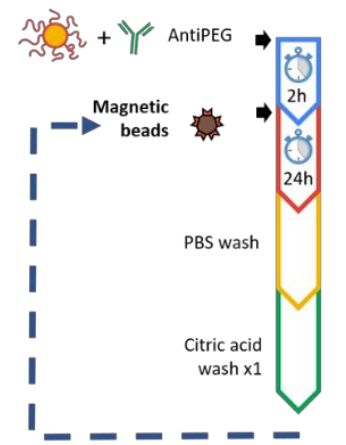

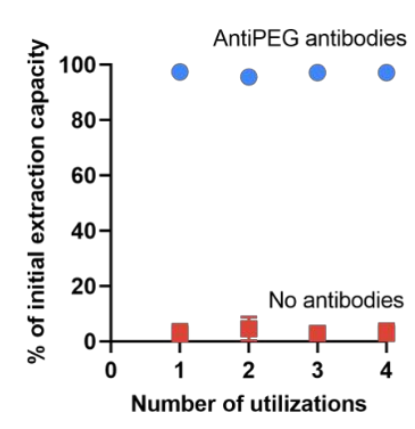

B. Reutilization of crosslinked magnetic beads in plasma
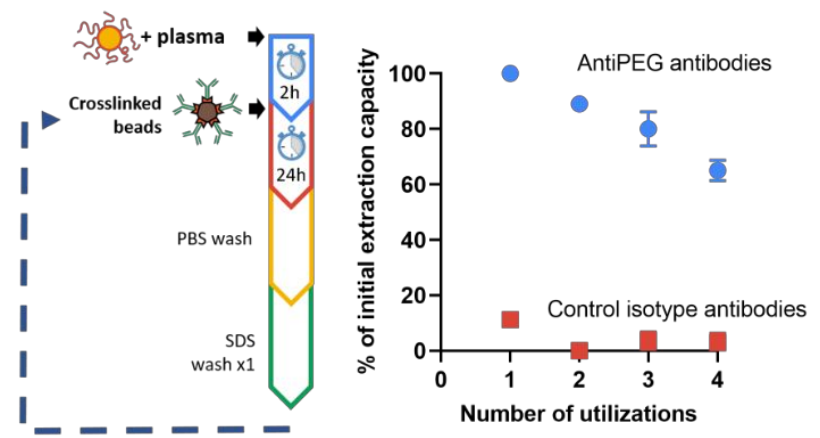

Figure S3. In PBS, magnetic beads can be reused without loss of capacity, but crosslinked magnetic beads in plasma become significantly less efficient upon reutilization. A. Nanoparticles are successfully extracted from PBS using non crosslinked 
magnetic beads, and magnetic beads do not lose their binding capacity after multiple extraction/elution cycles. B. Crosslinked magnetic beads lose approximately one third of their initial capacity after a full extraction/elution cycle using SDS $1 \%$. Values represent means $+/-S D(n=3)$. 
A. PEG-asparaginase (Oncaspar ${ }^{\circledR}$ ) extraction from PBS.

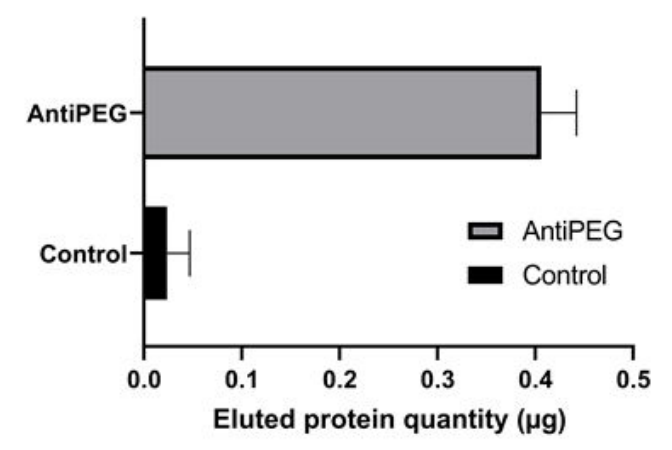

Figure S4. PEG-asparaginase can be selectively isolated using antiPEG magnetic beads, but with low capacity. The quantity of protein eluted from magnetic beads crosslinked to antiPEG antibodies or control antibodies after incubation with an excess of PEGylated asparaginase (Oncaspar尺) was evaluated by the bicinchonic acid (BCA) colorimetric assay. Values represent means $+/-\operatorname{SD}(n=3)$.

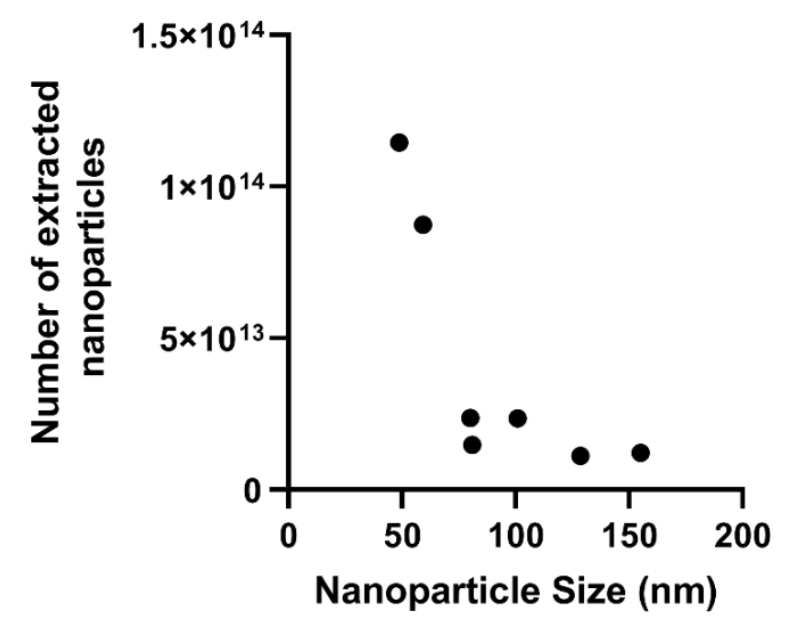

Figure S5. The number of PLGA-PEG nanoparticles which are extracted by $25 \mu \mathrm{L}$ of magnetic beads (equivalent to $750 \mu \mathrm{g}$ of solid) plotted against their average diameter. 
A. Centrifugation cannot quantitatively isolate PLGA-PEG nanoparticles and liposomes

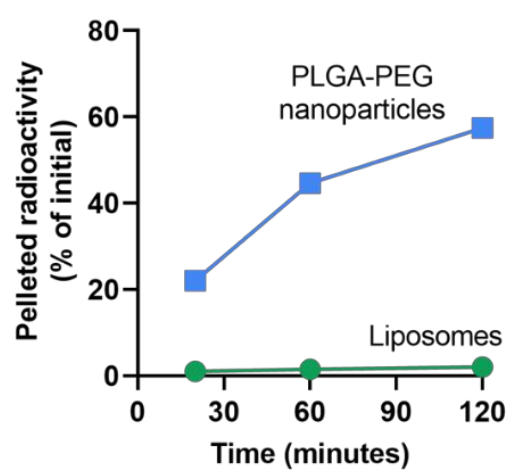

B. Effect of centrifugation on PLGA-PEG nanoparticles remaining in suspension

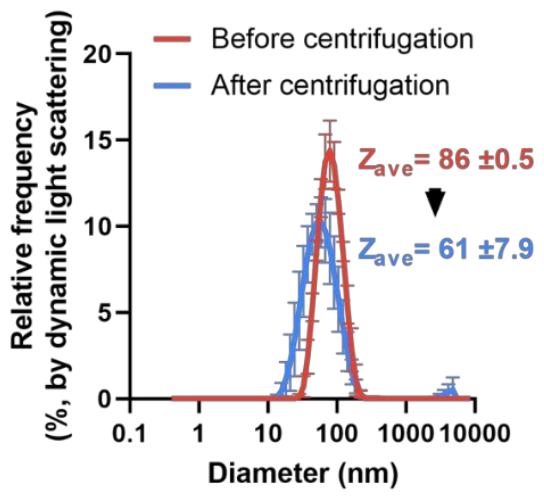

C. Extraction with magnetic beads followed by elution does not affect the size distribution

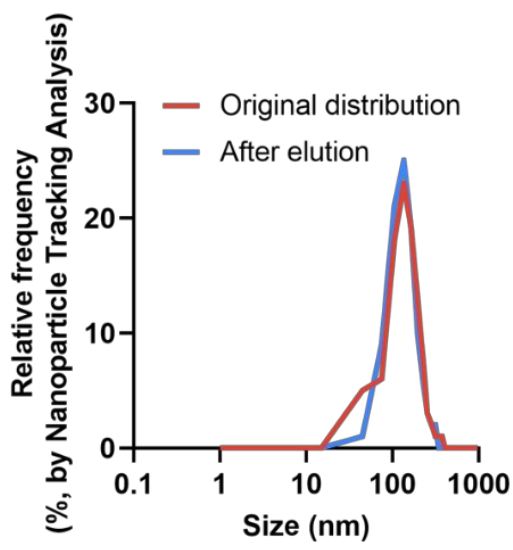

Figure S6. Extraction with magnetic beads is more efficient than centrifugation to isolate PLGA-PEG nanoparticles and liposomes A. Centrifugation at 15000 rcf for 120 minutes can result in the isolation of $60 \%$ of PLGA-PEG nanoparticles from plasma, but PEGylated liposomes cannot be isolated regardless of centrifugation time. Values represent means +/- SD ( $\mathrm{n}=3)$. B. In PBS, the size distribution of PLGA-PEG nanoparticles remaining in suspension after centrifugation (15,000 rcf $\times 120$ minutes) suggests an enrichment in smaller nanoparticles, suggesting that the isolated nanoparticles in the pellet mostly consist of the larger part of the distribution. C. In a single sample, immunoprecipitation from plasma followed by elution does not affect the size distribution of PLGA-PEG nanoparticles, as measured by FNTA. 

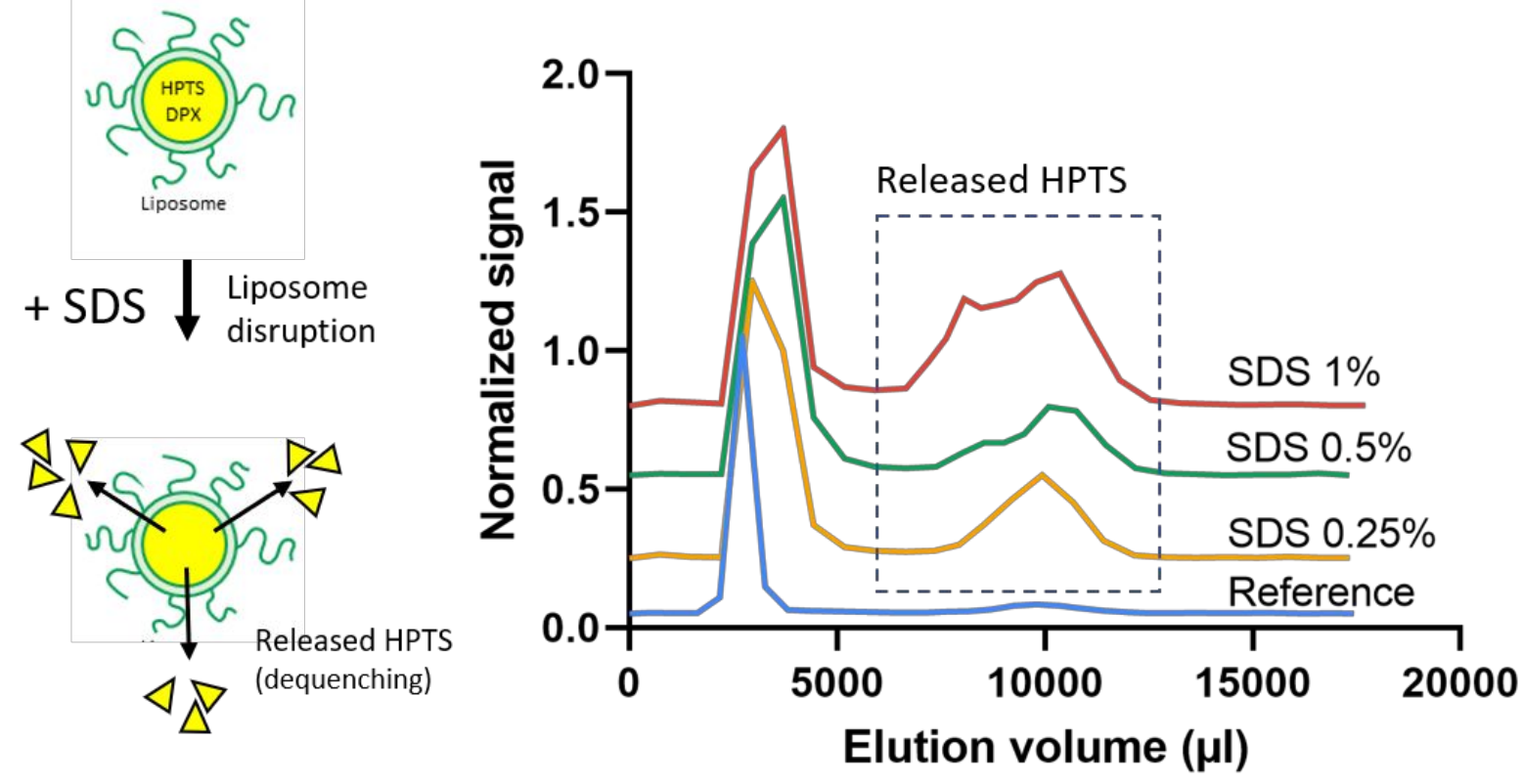

Figure S7. SDS triggers the release of the hydrophilic dye encapsulated in liposomes, suggesting that the surfactant disrupts the phospholipid membrane in a concentration dependent manner.

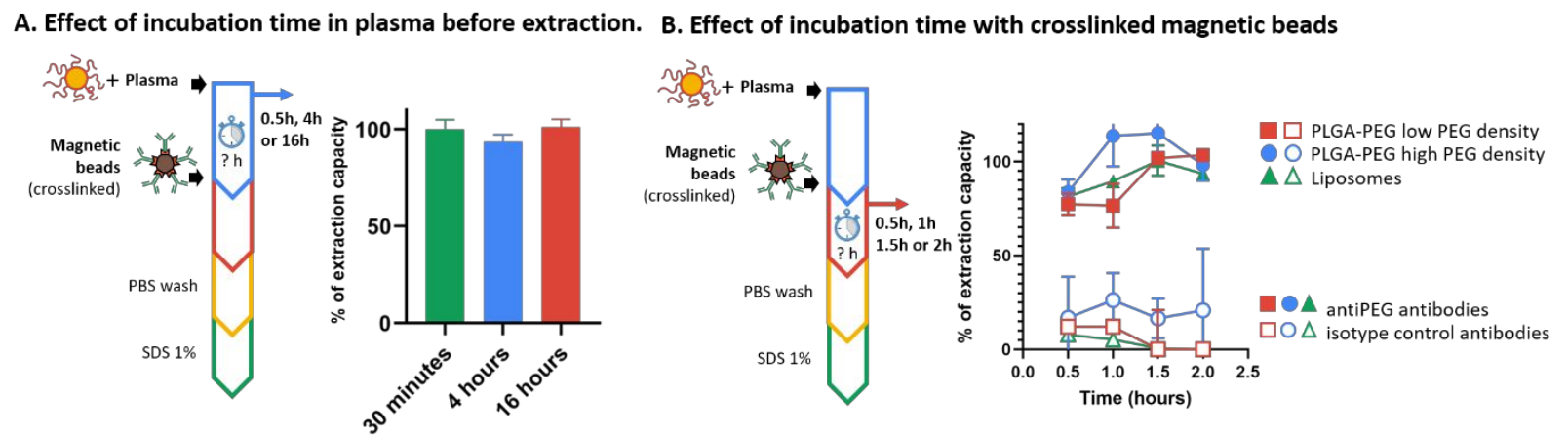

Figure S8. Effect of different incubation times on extraction capacity. A. Incubating nanoparticles in plasma before extraction does not alter performance of the immunoprecipitation. B. The kinetic of extraction of liposomes and PLGA-PEG nanoparticles reaches a plateau after 1.5 hours of incubation with magnetic beads. Values represent means $+/-S D(n=3)$ 


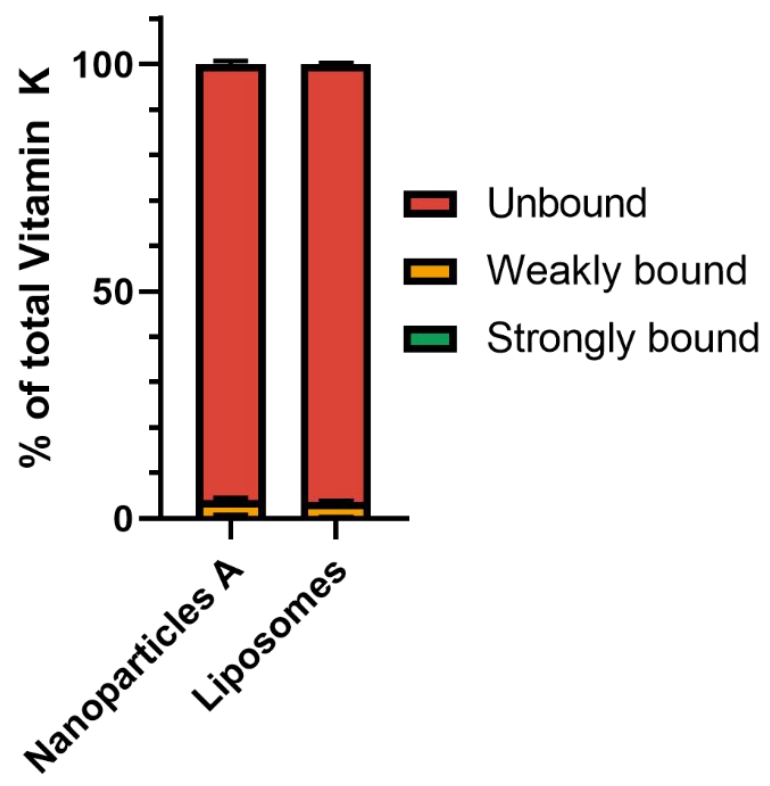

Figure S9. Drug-loaded nanoparticles and liposomes were incubated with magnetic beads crosslinked with control antibodies, supporting that Vitamin $\mathrm{K}$ isolated in SDS is encapsulated in PEGylated nanomedicines. Values represent means +/- SD $(n=3)$. 
Table S1. Type and characteristics of nanoparticles corresponding to each figure.

\begin{tabular}{|c|c|c|c|c|c|c|}
\hline $\begin{array}{l}\frac{T}{0} \\
\frac{\mathbb{Q}}{\bar{D}}\end{array}$ & $\begin{array}{l}0 \\
\frac{0}{3} \\
\frac{0}{8} \\
\frac{0}{\overline{7}} \\
\frac{0}{3}\end{array}$ & 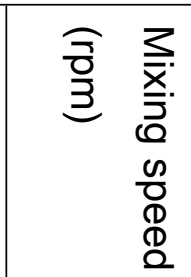 & 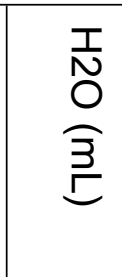 & 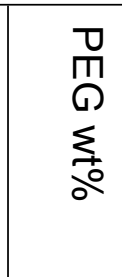 & 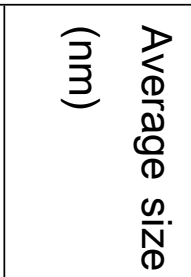 & 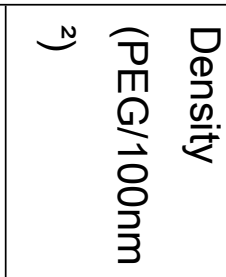 \\
\hline $1 \mathrm{~A}$ & $\begin{array}{l}50 \% \\
\text { 50\% }\end{array}$ & 1200 & 10 & $8 \%$ & 92 & 17 \\
\hline 1B & $\begin{array}{l}50 \% \\
\text { 50\% }\end{array}$ & 1200 & 10 & $8 \%$ & 200 & 38 \\
\hline 2 A B C & $\begin{array}{l}50 \% \\
\text { 50\% }\end{array}$ & 1200 & 10 & $8 \%$ & 101 & 19 \\
\hline $3 \mathrm{~A}$ & $\begin{array}{l}\text { PLGA20kPEG5 } \\
\mathrm{k}\end{array}$ & 1200 & 10 & $20 \%$ & 81 & 39 \\
\hline $3 \mathrm{~A}$ & $\begin{array}{l}60 \% \\
\text { PLGA27kPEG5 } \\
\mathrm{k} \\
40 \% \text { PLGA30k }\end{array}$ & 1200 & 10 & $8 \%$ & 103 & 20 \\
\hline $3 A$ & $\begin{array}{l}\text { Liposomes } \\
\text { (See materials } \\
\text { and methods) }\end{array}$ & I & 1 & 1 & 100 & 1 \\
\hline
\end{tabular}




\begin{tabular}{|c|c|c|c|c|c|c|}
\hline $3 B$ & $\begin{array}{l}55 \% \\
\text { PLGA10PEG5 } \\
45 \% \\
\text { PLGA28PEG5 }\end{array}$ & 1200 & 10 & $25 \%$ & 49 & 29 \\
\hline $3 B$ & $\begin{array}{ll}5 \% & \text { PLGA30k } \\
95 \% & \\
\text { PLGA28kPEG5 } \\
k\end{array}$ & 1200 & 10 & $14 \%$ & 59 & 21 \\
\hline $3 B$ & $\begin{array}{l}\text { PLGA20kPEG5 } \\
\mathrm{k}\end{array}$ & 1200 & 10 & $20 \%$ & 81 & 39 \\
\hline $3 B$ & $\begin{array}{l}60 \% \\
\text { PLGA27kPEG5 } \\
k \\
40 \% \text { PLGA30k }\end{array}$ & 1200 & 10 & $8 \%$ & 103 & 20 \\
\hline 3B & $\begin{array}{l}35 \% \\
\text { PLGA7kPEG5k } \\
65 \% \text { PLGA30k }\end{array}$ & 1200 & 10 & $15 \%$ & 129 & 45 \\
\hline 3B & 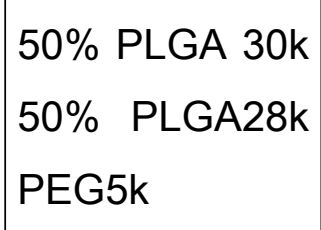 & 1200 & 10 & $8 \%$ & 85 & 16 \\
\hline 3B & $\begin{array}{l}40 \% \text { PLGA95k } \\
60 \% \\
\text { PLA21kPEG5k }\end{array}$ & 1200 & 10 & $11 \%$ & 155 & 41 \\
\hline $3 C$ & $\begin{array}{l}\text { Liposomes } \\
\text { (See materials } \\
\text { and methods) }\end{array}$ & I & I & I & 104 & 1 \\
\hline
\end{tabular}




\begin{tabular}{|c|c|c|c|c|c|c|}
\hline $3 D$ & $\begin{array}{lr}55 \% & \text { PLGA30k } \\
45 \% & \\
\text { PLGA7kPEG5k } \\
10 \% & \text { PLA- } \\
\text { CY5.5 } & \end{array}$ & 1600 & 10 & $19 \%$ & 126 & 56 \\
\hline $4 \mathrm{~A}$ & $\begin{array}{l}35 \% \\
\text { PLGA7kPEG5k } \\
65 \% \text { PLGA30k }\end{array}$ & 1200 & 10 & $15 \%$ & 121 & 44 \\
\hline $4 \mathrm{~A}$ & $\begin{array}{l}50 \% \\
\text { PLA21kPEG5k } \\
50 \% \text { PLGA95k }\end{array}$ & 1200 & 10 & $10 \%$ & 167 & 39 \\
\hline 4B & $\begin{array}{l}40 \% \text { PLGA95k } \\
60 \% \\
\text { PLA21kPEG5k }\end{array}$ & 1200 & 10 & $11 \%$ & 140 & 37 \\
\hline $4 C$ & $\begin{array}{l}40 \% \text { PLGA95k } \\
60 \% \\
\text { PLA21kPEG5k }\end{array}$ & 1200 & 10 & $11 \%$ & 140 & 37 \\
\hline $5 A$ & $\begin{array}{l}40 \% \text { PLGA95k } \\
60 \% \\
\text { PLA21kPEG5k }\end{array}$ & 1200 & 10 & $11 \%$ & 140 & 37 \\
\hline $5 B$ & $\begin{array}{l}20 \% \text { PLGA95k } \\
80 \% \\
\text { PLA21kPEG5k }\end{array}$ & 1600 & 8 & $4 \%$ & 126 & 12 \\
\hline
\end{tabular}




\begin{tabular}{|c|c|c|c|c|c|c|}
\hline $5 B$ & $\begin{array}{l}\text { Liposomes } \\
\text { (See materials } \\
\text { and methods) }\end{array}$ & I & I & 1 & 109 & 1 \\
\hline S1A & $\begin{array}{l}\text { PLGA20kPEG5 } \\
\mathrm{k}\end{array}$ & 1200 & 10 & $20 \%$ & 81 & 39 \\
\hline S1A & $\begin{array}{l}60 \% \\
\text { PLGA27kPEG5 } \\
\mathrm{k} \\
40 \% \text { PLGA30k }\end{array}$ & 1200 & 10 & $8 \%$ & 103 & 20 \\
\hline S1A & $\begin{array}{l}\text { Liposomes } \\
\text { (See materials } \\
\text { and methods) }\end{array}$ & I & I & 1 & 100 & 1 \\
\hline S1B & $\begin{array}{l}50 \% \text { PLGA 30k } \\
50 \% \text { PLGA28k } \\
\text { PEG5k }\end{array}$ & 1200 & 10 & $8 \%$ & 101 & 19 \\
\hline S1B & $\begin{array}{l}50 \% \text { PLGA95k } \\
50 \% \\
\text { PLA21kPEG5k }\end{array}$ & 800 & 10 & $11 \%$ & 147 & 41 \\
\hline $\mathrm{S} 2 \mathrm{~A}$ & $\begin{array}{l}\text { PLGA20kPEG5 } \\
\mathrm{k}\end{array}$ & 1200 & 10 & $20 \%$ & 81 & 39 \\
\hline S2B & $\begin{array}{l}50 \% \text { PLGA95k } \\
50 \% \\
\text { PLA21kPEG5k }\end{array}$ & 800 & 10 & $11 \%$ & 147 & 41 \\
\hline
\end{tabular}




\begin{tabular}{|c|c|c|c|c|c|c|}
\hline S3A & 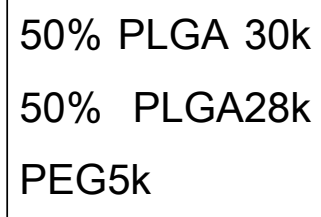 & 1200 & 10 & $8 \%$ & 92 & 17 \\
\hline S3B & $\begin{array}{l}50 \% \\
50 \%\end{array}$ & 1200 & 10 & $8 \%$ & 101 & 19 \\
\hline S5A & $\begin{array}{l}\text { Liposomes } \\
\text { (See materials } \\
\text { and methods) }\end{array}$ & I & 1 & 1 & 100 & 1 \\
\hline S5A & 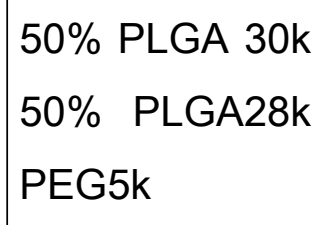 & 1200 & 10 & $8 \%$ & 85 & 16 \\
\hline S5B & 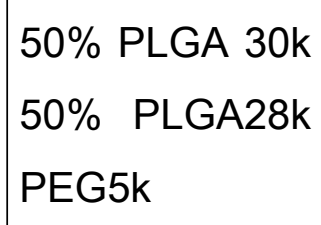 & 1200 & 10 & $8 \%$ & 85 & 16 \\
\hline S5C & $\begin{array}{l}\text { 36\% } \text { PLGA95k } \\
55 \% \\
\text { PLA21kPEG5k } \\
9 \% \\
\text { PLAP(R6G)- }\end{array}$ & 1200 & 10 & $11 \%$ & 155 & 41 \\
\hline S6 & $\begin{array}{l}55 \% \\
\text { PLGA10PEG5 } \\
45 \% \\
\text { PLGA28PEG5 }\end{array}$ & 1200 & 10 & $25 \%$ & 49 & 29 \\
\hline
\end{tabular}




\begin{tabular}{|c|c|c|c|c|c|c|}
\hline S6 & $\begin{array}{l}5 \% \text { PLGA30k } \\
95 \% \\
\text { PLGA28kPEG5 } \\
k\end{array}$ & 1200 & 10 & $14 \%$ & 59 & 21 \\
\hline S6 & $\begin{array}{l}\text { PLGA20kPEG5 } \\
\mathrm{k}\end{array}$ & 1200 & 10 & $20 \%$ & 81 & 39 \\
\hline S6 & $\begin{array}{l}60 \% \\
\text { PLGA27kPEG5 } \\
k \\
40 \% \text { PLGA30k }\end{array}$ & 1200 & 10 & $8 \%$ & 103 & 20 \\
\hline S6 & $\begin{array}{l}35 \% \\
\text { PLGA7kPEG5k } \\
65 \% \text { PLGA30k }\end{array}$ & 1200 & 10 & $15 \%$ & 129 & 45 \\
\hline S6 & $\begin{array}{l}50 \% \text { PLGA 30k } \\
50 \% \text { PLGA28k } \\
\text { PEG5k }\end{array}$ & 1200 & 10 & $8 \%$ & 85 & 16 \\
\hline S6 & $\begin{array}{l}36 \% \text { PLGA95k } \\
55 \% \\
\text { PLA21kPEG5k } \\
9 \% \text { BDP(R6G)- } \\
\text { PLA }\end{array}$ & 1200 & 10 & $11 \%$ & 155 & 41 \\
\hline S7A & $\begin{array}{l}50 \% \text { PLGA95k } \\
50 \% \\
\text { PLA21kPEG5k }\end{array}$ & 800 & 10 & $11 \%$ & 147 & 41 \\
\hline
\end{tabular}




\begin{tabular}{|l|l|l|l|l|l|l|}
\hline S7B & $\begin{array}{l}60 \% \\
\text { PLGA27kPEG5 } \\
\mathrm{k} \\
40 \% \text { PLGA30k }\end{array}$ & 1200 & 10 & $8 \%$ & 103 & 20 \\
\hline S8 & $\begin{array}{l}\text { Liposomes } \\
\text { (See materials } \\
\text { and methods })\end{array}$ & $/$ & $/$ & $/$ & 104 & $/$ \\
\hline S9 & $\begin{array}{l}20 \% \text { PLGA95k } \\
80 \% \\
\text { PLA21kPEG5k }\end{array}$ & 1600 & 8 & $4 \%$ & 126 & 12 \\
\hline S9 & $\begin{array}{l}\text { Liposomes } \\
\text { (See materials } \\
\text { and methods })\end{array}$ & $/$ & $/$ & $/$ & 109 & $/$ \\
\hline
\end{tabular}

[1] N. Bertrand, P. Grenier, M. Mahmoudi, E.M. Lima, E.A. Appel, F. Dormont, J.-M. Lim, R. Karnik, R. Langer, O. Farokhzad, Mechanistic understanding of in vivo protein corona formation on polymeric nanoparticles and impact on pharmacokinetics, Nat Commun, 8 (2017) 777.

[2] F. Brandl, N. Bertrand, E.M. Lima, R. Langer, Nanoparticles with photoinduced precipitation for the extraction of pollutants from water and soil, Nat Commun, 6 (2015) 7765.

[3] N. Bertrand, P. Simard, J.-C. Leroux, Serum-stable, long-circulating, pH-sensitive PEGylated liposomes, in: G.G. D'souza (Ed.) Liposomes: methods and protocols, Humana Press, Clifton, NJ, 2017, pp. 193-207.

[4] A. Dikpati, F. Mohammadi, K. Greffard, C. Quéant, P. Arnaud, G. Bastiat, I. Rudkowska, N. Bertrand, Residual Solvents in Nanomedicine and Lipid-Based Drug 
Delivery Systems: a Case Study to Better Understand Processes, Pharm Res, 37 (2020) 149.

[5] E. Chotard, F. Mohammadi, P. Julien, L. Berthiaume, I. Rudkowska, N. Bertrand, Drinkable lecithin vesicles to study the biological effects of individual hydrophobic macronutrients and food preferences, Food Chemistry, 322 (2020) 126736.

[6] I.M.d.O. Viana, P. Grenier, J. Defrêne, F. Barabé, E.M. Lima, N. Bertrand, Role of the complement cascade on the biological fate of liposomes in rodents, Nanoscale, 12 (2020) 18875-18884.

[7] Y. Ishihama, Y. Oda, T. Tabata, T. Sato, T. Nagasu, J. Rappsilber, M. Mann, Exponentially Modified Protein Abundance Index (emPAl) for Estimation of Absolute Protein Amount in Proteomics by the Number of Sequenced Peptides per Protein, Molecular \& Cellular Proteomics, 4 (2005) 1265-1272. 